\title{
Assisting inhabitants of residential homes with management of their energy consumption
}

\author{
Michael Kugler ${ }^{1}$, Elisabeth André ${ }^{1}$, Masood Masoodian ${ }^{2}$, Florian Reinhart ${ }^{3}$, \\ Bill Rogers ${ }^{2}$, and Kevin Schlieper ${ }^{3}$ \\ 1 Human Centered Multimedia \\ University of Augsburg \\ Augsburg, Germany \\ mail@michaelkugler.de, andre@informatik.uni-augsburg.de \\ 2 Department of Computer Science \\ The University of Waikato \\ Hamilton, New Zealand \\ masood, coms0108\}@cs.waikato.ac.nz \\ 3 Bottled Software GmbH \\ St. Leon-Rot, Germany \\ \{florian, kevin\}@bottledsoftware.de
}

\begin{abstract}
Although there are already a range of energy monitoring and automation systems available in the market that target residential homes, mostly with the aim of reducing their total energy consumption, very few of these systems are directly concerned with how those energy savings are actually made. As such, these systems do not provide tools that would allow users to make intelligent decisions about their energy usage strategies, and encourage them to change their energy use behaviour. In this paper we describe a system designed to facilitate planning and control of energy usage activities in residential homes. We also report on a user study of this system which demonstrates its potential for making energy savings possible.
\end{abstract}

Key words: Residential energy consumption, efficient energy usage, energy usage management, smart homes

\section{Introduction}

Energy consumed in private homes, particularly in developed countries, can constitute a large percentage of total energy consumption (e.g. 29\% in Europe [6]). This, combined with the fact that the worldwide energy consumption is increasing, has led to development of a range of technologies in recent years directed at reducing energy use in private households. These technologies aim to assist people by providing them with information about their energy usage, with the hope that this information will lead to them reducing the amount of energy they consume. However, the problem of saving energy use in residential homes is not just about reducing the total amount of energy consumed, but it is also 
about the better management of energy usage particularly at peak times (see $[3]$ ), which require a short-term energy production much higher than the daily average, as well as providing larger grid capacity by energy suppliers .

Furthermore, in an attempt to reduce reliance on fossil-fuel, more emphasis is currently being put on generation of electricity through renewable resources such as hydro, wind, and solar energy. Unlike traditional fossil-fuel or nuclear power plants, most renewable energy generation options are less predictable, and require more careful management.

Fortunately, although there are many tasks in a typical household which require use of energy, not all these tasks need to be carried out at specific times, but rather during certain time frames. For example, an electric car can generally be charged any time between the afternoon when the user arrives home and the next morning when the user leaves for work. Better management of these types of tasks could lead to use of energy when it is more readily available (e.g. off peek or when renewable energy is available).

To support the process of improving the management of energy use in private homes, it is important to identify where and how energy is being used by different devices in a household, and then provide tools which assist individual residents with changing their energy use behaviour.

Although there are now some home automation and energy monitoring devices in the market, most of these technologies only aim to allow users to reduce their total (e.g. daily, weekly, or monthly) energy use. Using these systems it is often very difficult to find out when and how much energy each device in a household is using, or if there are more than one person living in a house, how much energy each person consumes.

In this paper we briefly introduce a system we have developed, called USEM (Ubiquitous Smart Energy Management), which allows monitoring energy consumption by individual devices and inhabitants of private homes [8]. Here we focus more specifically on those mechanisms provided by USEM for controlling devices and scheduling tasks that consume energy, which would allow making energy savings in residential homes in an intelligent manner. We also briefly discuss a user study of USEM along with its related findings.

\section{Energy Monitoring and Home Automation Systems}

Existing technologies designed to assist users with saving energy in residential homes can be divided into two categories, those for monitoring energy consumption, and those for providing home automation capabilities.

Energy monitoring systems such as Current Cost ${ }^{4}$, the Energy Detective ${ }^{5}$ and Wattson ${ }^{6}$ generate energy consumption statistics, which users can then analyse to see where and how energy could be saved in the future. Although these systems are helpful to some extent, they often only give total energy use measurements

\footnotetext{
${ }^{4}$ http://www.currentcost.com

${ }^{5}$ http://www.theenergydetective.com

${ }^{6}$ http://www.diykyoto.com
} 
for the entire household, rather than providing details at the individual appliance level. Furthermore, these systems do not actively control appliances to save energy, neither do they make any suggestions to the user as to how energy savings could be made.

Home automation systems, on the other hand, are designed to actively control household appliances, as well as any other possible sources of energy use. Examples of such systems are HomeMatic ${ }^{7}$, Gira $^{8}$ and Intellihome ${ }^{9}$. Home automation systems are usually equipped with sensors (e.g. for temperature, motion detection, etc.), which are monitored by the system to allow it to react to environmental changes (e.g. someone enters a room, a window is left open, etc.) by controlling various actuators (e.g. for opening and closing doors and windows, turning lights on and off, etc.).

Unfortunately, despite their potential benefits for saving energy, home automation systems are not widely used in private homes. One of the main reasons for this is because home automation systems need to be able to control off-theshelf devices. However, at present there are no widely adopted home automation communication standards (e.g. $\mathrm{KNX}^{10}$ ). Due to this lack of communication standards most appliances cannot be effectively controlled by home automations systems, other than perhaps just turning them on or off. Although this level of control is sufficient for simple devices (e.g. lights), it is less than ideal for most devices which have programmable functionality (e.g. washing machines, dishwashers, ovens, etc.).

The other reason why home automation systems are not widely used is probably due to the complex configuration of such systems. Most people do not want to, or simply cannot deal with complex system set-up and operation required by home automation technology.

Several recent attempts have been made to overcome the shortcomings of energy monitoring and home automation systems by combining these two types of technologies to allow more intelligent monitoring and management of energy use in residential homes. These types of combined systems are viewed as being particularly useful for off-the-grid households where better management of energy use is much needed [2].

One such system is AIM [4] which combines measurement and automation approaches to reduce energy consumption. However, AIM requires the users to install specific hardware which can only control specific types of supported devices, and therefore, cannot be used to control existing off-the-shelf appliances.

A system that aims to support off-the-shelf devices is the Energy Aware Smart Home [7], designed to make energy usage more transparent by providing real-time energy consumption information at the device level. To measure energy consumption for each device, "Ploggs" socket adapters are used to transmit

\footnotetext{
7 http://www $\cdot h o m e m a t i c . c o m$

${ }^{8}$ http://www.gira.de

${ }^{9}$ http://www.intellihome.com

10 http://www.knx.org
} 
energy consumption data wirelessly via Bluetooth or ZigBee ${ }^{11}$ to a server. The consumption data is displayed to the user through a stationary interface, as well as a "UbiLense" augmented reality technology. The system focuses more on providing energy consumption data rather than allowing interaction with devices.

A similar system has been developed by Intel ${ }^{\circledR}$ as part of their Intelligent Home Energy ${ }^{12}$ management platform. This proof-of-concept system uses the ZigBee technology to communicate wirelessly with home appliances that are plugged into remote controllable power sockets. Although the system allows monitoring energy consumption, and some device control, it does not provide scheduling functionality to automatically manage execution of energy consuming household tasks.

Yet another example of a system that combines energy measurement and control hardware is the Home Energy Saving System (HESS) [5]. However, HESS only aims to reduce standby power consumption by switching off devices when they are not in use.

In summary, most of the above mentioned approaches either require the installation of special hardware, or provide very little control over energy using devices, other than perhaps turning them on and off, with no task scheduling functionality to allow automatic management of their use in an energy efficient manner.

\section{Requirements of an Automatic Control and Scheduling System}

As mentioned earlier, most existing residential home technology for energy usage monitoring and automation aim to assist users with reducing their total energy consumption, without caring too much about how those savings are made at the individual appliances level. This is because in most countries electricity is supplied to domestic users at a single rate, independent of when the electricity is used, or how it is generated. Although some differential rates (e.g. day and night time rates) may exist, these apply to only a certain range of energy using tasks (e.g. heating, hot water). This is however changing, as electricity suppliers aim to reduce peak time usage which is costly in terms of generation and the grid capacity they have to support. With the introduction of "smart metering" in an increasing number of residential homes in developed countries, users are given the option of changing their usage patterns to consume electricity when it is cheaper to supply.

Furthermore, as our reliance on renewable sources of energy, with their inherent variability, increases it becomes crucial to manage the use of energy intelligently so that energy is used when it is more readily available, and savings are

\footnotetext{
${ }^{11}$ http://www.zigbee.org

12 http://www.intel.com/p/en_US/embedded/applications/energy/energymanagement
} 
made when it is not. This is particularly important for houses that are off-grid and/or are reliant on their own renewable small-scale electricity generation (e.g. wind turbine, solar panel, etc.).

In this paper we propose an intelligent system that allows users to specify their preferences, in the form of rules, tasks and levels, which can then be used to automatically control how and when home appliances are used by scheduling household energy usage activities. To do this, we identify two categorises of appliances:

- Regular devices are used to perform specific individual tasks that have a set duration. Most home appliances (e.g. dish washer, TV, oven, etc.) fall into this category, and can have two or more modes of operation. Simple devices are either on or off (e.g. lights), while more advanced devices have many different modes of operation (e.g. a washing machine can be on, off, on standby, in colour wash or warm wash mode, etc.).

- Continuous devices operate more automatically without much manual control by the user. Devices such as a hot water heater, air conditioner, refrigerator, etc. fall into this category. These devices do not generally have an operating mode like regular devices do, but rather try to automatically maintain a value (e.g. water or air temperature) within a user specified range.

For regular devices we define rules and tasks as:

- A rule has a set of conditions, that once met, allow the rule to be executed to cause some effect. There are a range of conditions that the system can check (e.g. weather conditions, presence of people, energy prices, etc.). The effect of a rule being executed is usually to switch one or more regular devices to a specified operating mode. Rules allow users to configure the system to automatically perform reoccurring tasks (e.g. turn the lights off if nobody is at home).

- A task is a one off activity that the user gets the system to schedule and perform. Tasks have a number of variables which the user can specify. For example, the operating mode in which the device should run, for how long, etc. As with rules, execution of tasks can also be dependant on certain conditions being met (e.g. the washing is done only if there is someone at home).

For continuous devices, which automatically maintain a value within a user specified range, rather than defining rules and tasks we define levels.

- A level is similar to a rule, in that it allows setting conditions for continuous devices. In addition to all the conditions available for rules, a time condition can also be set for a level. This makes it possible, for instance, to set a level which tells the system to reduce heating to a lower temperature to if nobody is at home on weekdays between 8a.m and 4p.m. 


\section{Controlling Appliances and Scheduling Tasks in USEM}

We have developed a prototype system called USEM [8] to allow monitoring and control of energy usage in residential homes. The system provides interfaces for smart phones and tablets which can be used to control and monitor energy use while mobile. The system also provides a web interface for setting rules, tasks, and levels for controlling regular and continuous devices. In this section we describe the web interface of USEM.

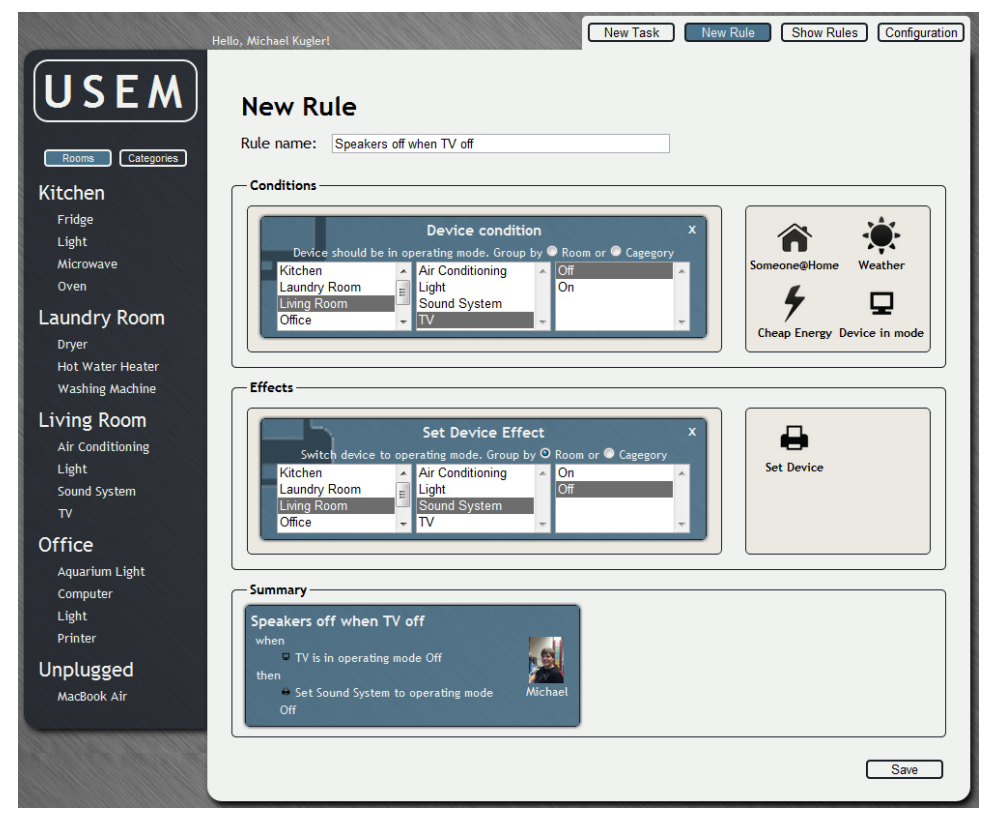

Fig. 1. The Rule Editor of the web interface component of USEM.

Figure 1 shows the Rule Editor component of USEM which can be used to create new rules, or edit the existing ones. The interface allows the user to choose conditions and effects from a set of templates (shown on the right), for devices that are connected to the system. After adding conditions and effects the user can then set further parameters (e.g. a temperature range for the weather condition). The interface displays a summary of the rule which will be created at the bottom. Users can also view all the rules they have created. To make finding existing rules easier, the rules are grouped by their conditions (e.g. Weather, Someone@Home, etc.), and the parameters for these conditions (e.g. rainy, cloudy, etc. for the Weather condition).

The Task Editor component of USEM, shown in Figure 2, enables users to create new tasks, or edit the existing ones. Users can choose a device, and an affiliated operating mode, for which they want to create a task. They can then 


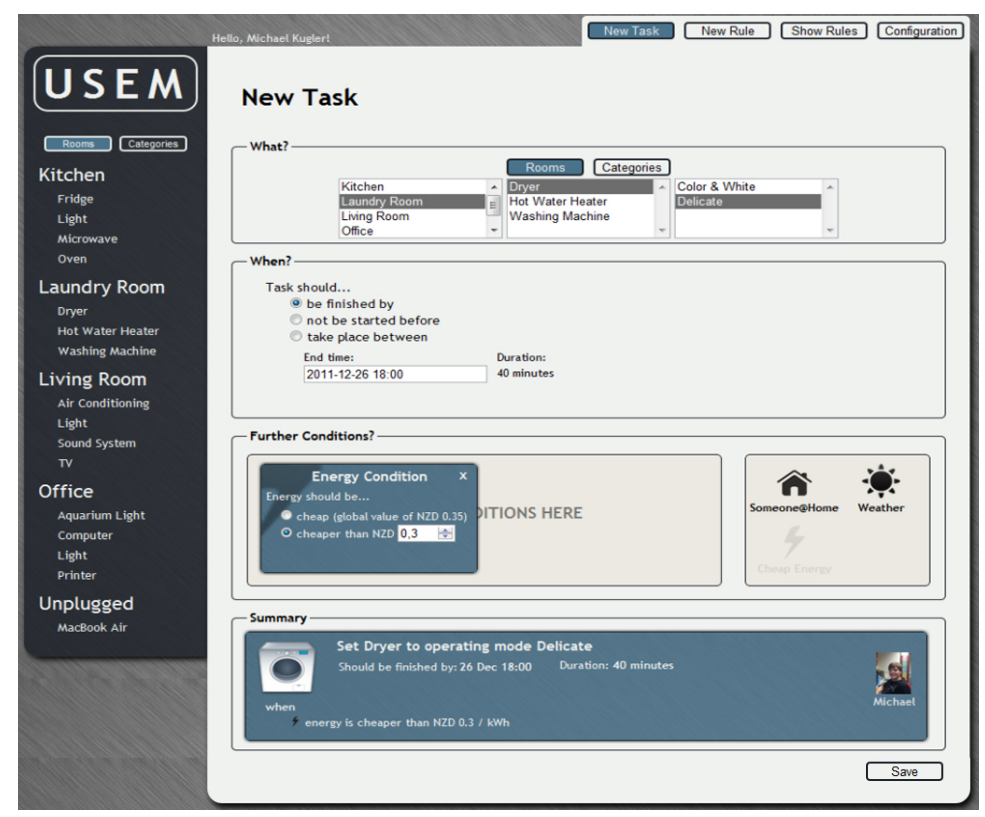

Fig. 2. The Task Editor of the web interface component of USEM.

specify the time boundaries for the task (e.g. when the tasks should be finished by, a time before which the task should not start, or a timespan in which the task should be executed). Users can also add conditions to the task in a manner similar to that of the Rule Editor. The web interface provides a summary of the task which is going to be created at the bottom.

Once a task has been created, USEM attempts to schedule the task, based on the conditions set by the user, including the time boundaries, etc. The user can view all the scheduled tasks (see Figure 3) using a visualization based on glyphs [1]. The user defined time boundaries of a task are shown in a gray box in the background, and the actual planned execution time by the system is shown as a colored box in the foreground. A color range of green to red is used to represent the amount of renewable energy which is likely to be available when the task starts, based on the estimation provided by USEM. The amount of energy required for the task is shown by a speedometer icons (e.g. less than $0.5 \mathrm{kWh}$ ). These are obtained from a device profile database maintained by the system.

USEM uses the JBoss Drools Expert ${ }^{13}$ framework to manage the execution of all its rules. The task scheduling and optimization is done by using the JBoss Drools Planner ${ }^{14}$ component. Rules and tasks are directly converted into the Drools Rule Language (DRL) format, readable by these components.

13 http://www.jboss.org/drools/drools-expert

14 http://www.jboss.org/drools/drools-planner 


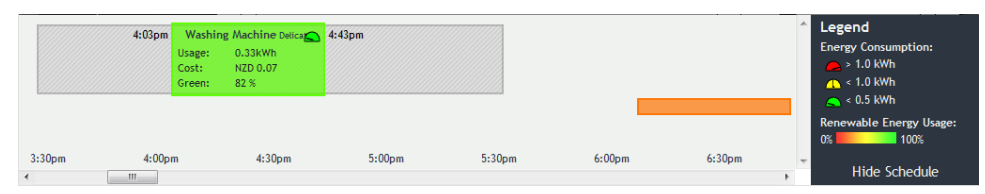

Fig. 3. An example schedule with detailed information shown for a selected task.

\section{User Evaluation}

We conducted a user study to evaluate the effectiveness of the various interfaces provided by USEM. In particular we aimed to see how easy the users would find the concepts of rules and tasks, and the process of setting up rules and scheduling tasks in USEM.

Twenty people participated in this study. They were 15 males, 5 females; 11 were students, 2 academics, 1 teacher and 6 others; aged 20 to 62 years old with an average age of 35 . All of the participants used computers on a daily basis.

\subsection{Methodology}

Each study session started with a short tutorial describing the functionality of the interface to be used. The participants were then given sufficient time to familiarise themselves with the system, before performing the actual study tasks. Each task was described in a couple of sentences, followed by several steps which the participants had to perform using the system.

The participants carried out four tasks using the web interface. Task 1 was to create a new rule; Task 2 was to set up a level for a continuous device; Task 3 was to create a new task for a regular device; and Task 4 was to interpret a schedule with several upcoming tasks.

After performing each task, the participants were required to answer two questions:

1. How easy was it to perform this task?

2. How useful do you find the functionality?

At the end of the session the participants were asked to complete a final questionnaire, with the following questions:

1. How easy would it be for you to adapt to using USEM for tasks, where you do not have to change your daily routine very much? (e.g. create tasks for doing the laundry, instead of just switching the washing machine on manually?)

2. Would you adapt your daily routine in order to use more renewable energy? (e.g. start cooking dinner an hour later?)

3. How useful do you find the overall system with regard to efficient energy usage?

A Likert scale of 1 (not easy/not useful) to 7 (very easy/very useful) was used for each of the questions used after the tasks and in the final questionnaires. 


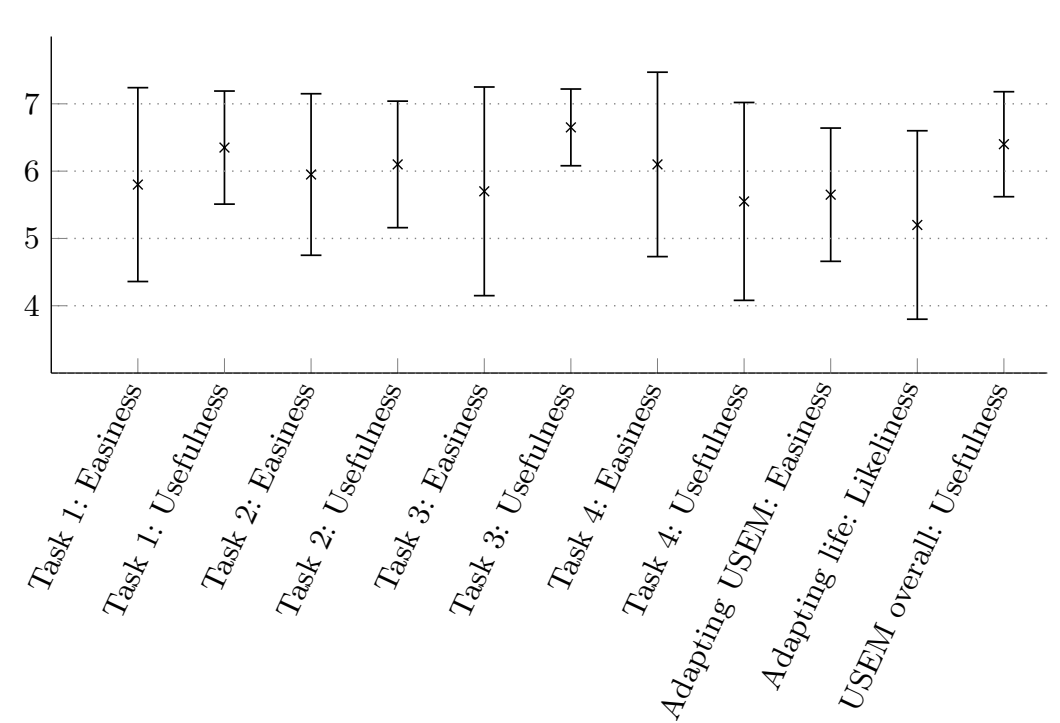

Fig. 4. A summary of the results of the questionnaires used in the study.

\subsection{Results and Discussion}

A summary of the results of the questionnaires used in the study is shown in Figure 4. Generally the participants rated all the four tasks as being easy to perform, with an average rating between 5.7 (for Task 3) and 6.1 (for Task 4). In terms of the usefulness of the functionality provided by USEM, the ratings were once again very positive, ranging on average between 5.7 (for Task 4) and 6.7 (for Task 3).

In response to the first question of the final questionnaire the participants gave an average rating of 5.7. This means that they believe it would be easy to adapt to using USEM for scheduling their tasks, rather than just doing them when they want a task to be done (e.g. asking USEM to schedule and do the laundry when it is cheaper rather than doing it right now). Of course unless a long-term evaluation of USEM is carried out, one cannot be sure that this is indeed what users will adapt to do.

Even in response to the question of whether the participants would adapt their daily routine in order to use more renewable energy, they gave an average rating of 5.2. This means that our participants were in favour of consuming renewable energy, and would schedule their tasks to be done when there is more renewable energy available. However, once again this is only their opinion, and may or may not lead to actual change of behaviour.

Overall, the usefulness of USEM with regard to efficient energy usage was rated 6.4. This indicates that our participants believe USEM could assists them in using energy more efficiently. 


\section{Conclusions}

This paper has described some of the components of USEM, which have been designed to facilitate scheduling and control of energy consuming tasks in residential homes. The results of a user study conducted to evaluate USEM demonstrate its potential benefits for assisting users in making energy saving tasks easy and useful. However, as we have pointed out, this study has only obtained users' opinions on the perceived usefulness of USEM. Therefore, a long-term study of USEM in real-life is still needed to confirm whether these results translate into actual practice, and if USEM would help users change their behaviour to save energy more intelligently.

\section{Acknowledgements}

We would like to thank the participants of our user study. This research is related to the IT4SE project, funded by BMBF. For more information visit http://www.it4se.net.

\section{References}

1. Aigner, W., Miksch, S., Thurnher, B., Biffl, S.: PlanningLines: novel glyphs for representing temporal uncertainties and their evaluation. In: Proceedings of the 9th International Conference on Information Visualisation. pp. 457-463. IV 2005 (2005)

2. Banerjee, N., Rollins, S., Moran, K.: Automating energy management in green homes. In: Proceedings of the 2nd ACM SIGCOMM workshop on Home networks. pp. 19-24. HomeNets 2011, ACM (2011)

3. Brown, R., Koomey, J.: Electricity use in California: past trends and present usage patterns. Energy Policy 31(9), 849-864 (2003), http://linkinghub.elsevier.com/ retrieve/pii/S0301421502001295

4. Capone, A., Barros, M., Hrasnica, H., Tompros, S.: A New Architecture for Reduction of Energy Consumption of Home Appliances. In: TOWARDS eENVIRONMENT, European conference of the Czech Presidency of the Council of the EU (2009), http://www.e-envi2009.org/presentations/S3/Barros.pdf

5. Choi, K., Ahn, Y., Park, Y., Park, W., Seo, H., Jung, K., Seo, K.: Architectural design of home energy saving system based on realtime energy-awareness. In: Proceedings of the 4th International Conference on Ubiquitous Information Technologies \& Applications. pp. 1-5. ICUT 2009, IEEE (2009)

6. European Union: Energy - Yearly statistics 2008. Yearly statistics (2010), http://epp.eurostat.ec.europa.eu/cache/ITY\_OFFPUB/KS-PC-10-001/EN/ KS-PC-10-001-EN.PDF

7. Jahn, M., Jentsch, M., Prause, C., Pramudianto, F., Al-Akkad, A., Reiners, R.: The energy aware smart home. In: Proceedings of the 5th International Conference on Future Information Technology. pp. 1-8. FutureTech 2010 (may 2010)

8. Kugler, M., Reinhart, F., Schlieper, K., Masoodian, M., Rogers, B., André, E., Rist, T.: Architecture of a ubiquitous smart energy management system for residential homes. In: Proceedings of the 12th Annual Conference of the New Zealand Chapter of the ACM SIGCHI. pp. 101-104. CHINZ 2011 (2011) 